Detrended fluctuation analysis of scaling crossover effects

This article has been downloaded from IOPscience. Please scroll down to see the full text article.

2010 EPL 9010009

(http://iopscience.iop.org/0295-5075/90/1/10009)

View the table of contents for this issue, or go to the journal homepage for more

Download details:

IP Address: 24.40.207.50

The article was downloaded on 02/06/2011 at 16:55

Please note that terms and conditions apply. 


\title{
Detrended fluctuation analysis of scaling crossover effects
}

\author{
M. IgnaCCOLO ${ }^{1}$, M. LAtKa ${ }^{2(a)}$ and B. J. West ${ }^{3}$ \\ ${ }^{1}$ Physics Department, Duke University - P.O. Box 90319, Durham, NC 27r08-0319, USA \\ ${ }^{2}$ Institute of Biomedical Engineering, Wroclaw University of Technology - Wybrzeze Wyspianskiego 27, \\ 50-370 Wroclaw, Poland, EU \\ ${ }^{3}$ Mathematics Division, Army Research Office - P.O. Box 12211, Research Triangle, NC 27r09-2211, USA
}

received 26 January 2010; accepted in final form 26 March 2010 published online 4 May 2010

PACS 05.40.-a - Fluctuation phenomena, random processes, noise, and Brownian motion PACS 05.10.Gg - Stochastic analysis methods (Fokker-Planck, Langevin, etc.) PACS 87.10.Mn - Stochastic modeling

\begin{abstract}
Detrended fluctuation analysis (DFA) is one of the most frequently used fractal time series algorithms. DFA has also become the tool of choice for analysis of the short-time fluctuations despite the fact that its validity in this domain has never been demonstrated. We adopt an Ornstein-Uhlenbeck Langevin equation to generate a time series which exhibits short-time powerlaw scaling and incorporates the fundamental property of physiological control systems - negative feedback. To determine the scaling exponent, we derive the analytical expressions for the standard deviation of the solution $X(t)$ of this equation using both the ensemble of statistically independent trajectories and the ensemble obtained by partitioning a single trajectory. The latter approach is used in DFA and many other physiological applications. Surprisingly, the formulas for the standard deviations are different for these two ensembles. We demonstrate that the partitioning amounts to building up deterministic trends that satisfy the "trend + signal" decomposition assumption which is characteristic of DFA. Consequently, the dependence of the rms of DFA residuals $F(\tau)$ on the length $\tau$ of data window is the same for both ensembles. The growth of $F(\tau)$ is significantly different from that of the standard deviation of $X(t)$. While the DFA estimate of the shorttime scaling exponent is correct, the polynomial detrending delays the approach of $F(\tau)$ to the asymptotic value by as much as an order of magnitude. This delay may underlie the gradual change of the DFA scaling index typically observed in time series that exhibit crossover between the short- and long-time scaling.
\end{abstract}

editor's choice Copyright (c) EPLA, 2010

Detrended fluctuation analysis (DFA) [1] is one of the most frequently used algorithms for fractal analysis of experimental time series. Bashan et al. [2] mention that there are about 500 papers on DFA. This method was developed to improve on the original scaled window variance analysis of Mandelbrot [3,4]. In particular, DFA allows one to detect long-time, power-law scaling of the second moment of the time series fluctuations in the presence of additive, polynomial non-stationarities. Physiological time series, such as electroencephalogram (EEG), electrocardiogram (ECG), waveforms of arterial blood pressure (ABP) and cerebral blood flow velocity are notoriously non-stationary, which was, in part, the reason for the rapid adoption of this algorithm. The initial application of DFA to heart rate variability, namely to inter-beat interval time series, revealed the existence of two scaling

\footnotetext{
(a) E-mail: Miroslaw.Latka@pwr.wroc.pl
}

regimes with the crossover taking place at approximately 10 heart beats [5]. Interestingly enough, the short-time scaling exponent turned out to be clinically significant. For example, this measure was the most accurate predictor of all-cause mortality in a cohort of 446 survivors of acute myocardial infarction [6]. The "two exponent" approach was used to quantify heart rate variability in various physiological conditions [7-11], dynamics of arterial blood pressure $[12,13]$ and cerebral blood flow [14]. This prompts the question: Is DFA applicable in the short-time limit? Before we pose a second question, let us briefly describe the DFA algorithm.

Given a time series $\left\{\xi_{j}\right\}_{j=1}^{N}$, the zero-centered time series is aggregated:

$$
Z_{k}=\sum_{j=1}^{k}\left[\xi_{j}-\xi_{\text {avg }}\right], \quad k=1,2, \ldots, N,
$$


where $\xi_{\text {avg }}=(1 / N) \sum_{j=1}^{N} \xi_{j}$. The aggregated signal $\left\{Z_{k}\right\}$ is divided into $n_{t}$ non-overlapping windows of size $\tau\left(n_{t}=\right.$ $[N / \tau])$. For each window $l=1, \ldots, n_{t}$, a least-squares polynomial fit $y_{l}^{(m)}(k)$ of order $m$ is determined. The fitted trend is subtracted from the aggregated time series and the root mean square $F_{l}^{(m)}(\tau)$ of residuals $\tilde{Z}_{k, l}=$ $Z_{k, l}-y_{l}^{(m)}(k)$ is calculated:

$$
F_{l}^{(m)}(\tau)=\sqrt{\frac{1}{\tau} \sum_{k=1}^{\tau} \tilde{Z}_{k, l}^{2} .}
$$

After averaging $F_{l}^{(m)}$ over all $n_{t}$ windows, we obtain the mean value $F^{(m)}$. These steps are repeated for increasing values of the window size $\tau[1,15]$. One may also calculate $F^{(m)}(\tau)$ for sliding windows, an option that is available in the original implementation of DFA (http://www . physionet.org) [16].

The DFA assumes [2] the time series $\left\{Z_{k}\right\}$ to be the superposition of two independent contributions: the polynomial trend $T$ and the signal $S$

$$
Z_{k}=T_{k}+S_{k}
$$

with the null covariance $(\operatorname{Cov}(T, S)=0)$. However, the outcome of DFA does not by itself allow for the validation of the decomposition assumption eq. (3). Thus, a second question arises: Do physiologically relevant dynamic models violate the assumption of statistical independence of signal and trend?

The phenomenal pace of adoption of fractal time series analysis may be, to a large extent, attributed to the existence of a simple, solvable model — fractional Brownian motion (fBm) [17]. fBm has been invaluable not only in evaluating the performance of numerous scaling detection algorithms $[4,15]$ but also in establishing the connection between, for example, the growth of the second moment of dynamical variables and long-range correlations. The existence of long-range correlations in physiological time series could not be easily reconciled with the concept of homeostasis which over one hundred years earlier was recognized as the cornerstone of physiology. Homeostasis refers to the organism's ability to suppress fluctuations induced by changes in the environment and to return to equilibrium. The opposition between homeostasis and long-term memory fueled the interest in fractal methods $[18,19]$.

To answer our two questions concerning the applicability of DFA to physiological data, we adopt a simple stochastic model which exhibits short-time power-law scaling and incorporates a fundamental property of physiological control systems - negative feedback. This model, known in physics literature as the Ornstein-Uhlenbeck (OU) Langevin equation, will serve as a DFA testbed. Let us recall that the OU Langevin equation for a linearly dissipative stochastic process reads [20]:

$$
\frac{\mathrm{d} X(t)}{\mathrm{d} t}=-\lambda X(t)+\eta(t),
$$

where $\lambda$ is the dissipation rate. In the above equation, a zero-centered Gaussian random force $\eta(t)$ is delta correlated in time:

$$
\langle\eta(t) \eta(t+\tau)\rangle=\sigma_{\eta}^{2} \delta(\tau)
$$

$X(t)$ is also a Gaussian random process with a spectrum [21]:

$$
G(f)=\frac{2 \sigma_{\eta}^{2}}{\lambda^{2}+4 \pi^{2} f^{2}} .
$$

Consequently, from the convolution theorem one obtains the autocorrelation function of $X(t)$

$$
\rho(t)=e^{-\lambda t}
$$

$X(t)$ may be expressed as the formal solution to the firstorder, linear differential equation (4)

$$
X(t)=e^{-\lambda t}\left[X(0)+\int_{0}^{t} \eta\left(t^{\prime}\right) e^{\lambda t^{\prime}} \mathrm{d} t^{\prime}\right] .
$$

In the context of physiological time series analysis, we refer to an average $\langle\ldots\rangle$ over an ensemble of statistically independent realizations of eq. (4) as a multiple trajectory ensemble (MTE) average. Let us consider first the MTE of trajectories all starting from zero. Using eq. (8) we obtain the expression for the variance of $X$

$$
\sigma_{M}^{2}(t) \equiv\left\langle X^{2}(t)\right\rangle_{M}-\langle X(t)\rangle_{M}^{2}=\frac{\sigma_{\eta}^{2}}{2 \lambda}\left[1-e^{-2 \lambda t}\right] .
$$

Consequently, for $t \ll 1 / \lambda$ the variance increases linearly with time $\lim _{t \rightarrow 0} \sigma_{M}^{2}(t) \approx \sigma_{\eta}^{2} t$ and the short-time scaling index for the OU Langevin equation is 0.5 . See $[18,22]$ for a review of scaling in the natural sciences and medicine. For $t \gg 1 / \lambda$ the variance becomes time independent $\lim _{t \rightarrow \infty} \sigma_{M}^{2}(t)=\sigma_{\eta}^{2} /(2 \lambda)$ with a saturation induced by the dissipation. This saturation precludes asymptotic scaling of the OU Langevin model.

However, physiological data do not allow for MTE averaging. In applications, expectation values are calculated by partitioning a single experimental time series into an ensemble of data segments of equal length. For brevity, we call such an approach single trajectory ensemble (STE) averaging. The equivalence of averages calculated using these two ensembles had been assumed by Wang and Uhlenbeck as early as 1945 but not proven [21].

The comparison of both types of averaging is presented in fig. 1, where the standard deviation of the solution $X$ of the Langevin equation (4) is plotted as a function of the length $\tau$ of the data window. The MTE averaging was done using $n_{M}=5000$ trajectories of length $N_{M}=1000$ originating at zero and generated by numerical integration of eq. (4) with constant time step $\Delta t=1(\lambda=0.055$ and $\left.\sigma_{\eta}=40\right)$. The STE was created by partitioning a single trajectory of length $N_{S}=n_{M} N_{M}$ into non-overlapping segments of length $\tau$. It is rather surprising that the 


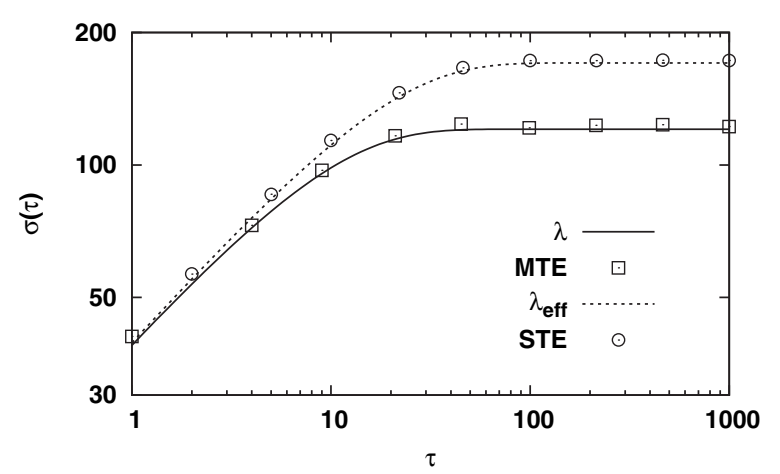

Fig. 1: Standard deviation of the solution $X$ eq. (8) is plotted as a function of the length of the data window $\tau(\lambda=0.055$ and $\left.\sigma_{\eta}=40\right)$. The numerical estimate of standard deviation was calculated using either the multiple (squares) or single (circles) trajectory ensembles. The theoretical value of the standard deviation is drawn for the MTE (solid line, cf. eq. (9)) and the STE (dashed line, cf. eq. (15)).

MTE and STE estimates of the standard deviation do not coincide.

To elucidate the difference shown in fig. 1, we derive the expression for the standard deviation of the solution $X(t)$ (8) using the STE. Please note that STE averaging involves relative displacements: $Z(t, \tau)=X(t+\tau)-X(t)$, $t \in[0, T-\tau]$. Using eq. (8), we can write $X(t+\tau)$ as

$$
X(t+\tau)=e^{-\lambda \tau}\left[X(t)+\int_{0}^{\tau} \eta\left(t+t^{\prime}\right) e^{\lambda t^{\prime}} \mathrm{d} t^{\prime}\right]
$$

and express $Z(t, \tau)$ in the following form:

$$
Z(t, \tau)=e^{-\lambda \tau} \int_{0}^{\tau} \eta\left(t+t^{\prime}\right) e^{\lambda t^{\prime}} \mathrm{d} t^{\prime}+X(t)\left[e^{-\lambda \tau}-1\right] .
$$

Please recall that the random force $\eta$ is stationary and consequently the time translation of $\eta$ in the integrand in eq. (11) does not affect the statistical properties of $Z(t, \tau)$. Without loss of generality, we may assume that a trajectory $X(t)$ starts at zero and then $Z(t, \tau)$ may be written in a particularly illuminating form:

$$
Z(t, \tau)=X(\tau)+X(t)\left[e^{-\lambda \tau}-1\right]
$$

The second term on the r.h.s. of eq. (12) makes $Z(t, \tau)$ explicitly time dependent. Thus, the properties of data segments of length $\tau$ generated by partitioning of a single trajectory are not identical (please note that in eq. (12) $t$ denotes the left endpoint of such data segments). In particular, $Z(t, \tau)$ being the sum of Gaussian variables is a Gaussian variable itself with zero mean value $(E[Z(t, \tau)]=0)$ and the following variance:

$$
E\left[Z^{2}(t, \tau)\right]=\sigma_{M}^{2}(\tau)+\left[e^{-\lambda \tau}-1\right]^{2} \sigma_{M}^{2}(t)
$$

The variance $\sigma_{S}^{2}(\tau)$ for the single trajectory ensemble is just $E\left[Z^{2}(t, \tau)\right]$ time averaged along the trajectory

$$
\begin{aligned}
\sigma_{S}^{2}(\tau) & =\frac{1}{T-\tau} \int_{0}^{T-\tau} E\left[Z^{2}(t, \tau)\right] \mathrm{d} t \\
& =\sigma_{M}^{2}(\tau)+\frac{\left(e^{-\lambda \tau}-1\right)^{2} \sigma_{\eta}^{2}}{2 \lambda}\left[1+\frac{e^{-2 \lambda(T-\tau)}-1}{2 \lambda(T-\tau)}\right],
\end{aligned}
$$

where $T$ is the length of the trajectory. Taking into account that $\lambda T \gg 1$, we obtain the following approximation:

$$
\sigma_{S}^{2}(\tau) \approx \frac{\sigma_{\eta}^{2}}{\lambda}\left(1-e^{-\lambda \tau}\right)
$$

Thus, the variance for the single trajectory ensemble is given by the formula eq. (9) for the MTE, albeit with the effective dissipation rate $\lambda_{e f f}=\lambda / 2$ which is half that of the MTE. Both curves $\sigma_{M}(\tau)$ and $\sigma_{S}(\tau)$ are plotted in fig. 1 and are in agreement with the relevant numerical calculations. It is apparent from eqs. (9) and (15) that the choice of the ensemble does not affect the value of the short-time scaling exponent, which in both cases is equal to $1 / 2$.

In the classic paper Wang and Uhlenbeck wrote [21]: "... One can then cut the record in pieces of length $\mathrm{T}$ (where $\mathrm{T}$ is long compared to all periods occurring in the process), and one may consider the different pieces as the different records of an ensemble of observations. In computing average values one has in general to distinguish between an ensemble average and a time average. However, for a stationary process these two ways of averaging will always give the same result ...".

This statement, given without a formal proof, has implicitly but erroneously linked the problem of validity of phenomenological (STE) ensembles to the ergodicity of the underlying dynamical system. The discrepancy between $\sigma_{S}$ and $\sigma_{M}$ clearly demonstrates that the assumption of equivalency of STE and MTE averages may be false even for a stationary and ergodic model such as the OU Langevin equation (4). Please note that the asymptotic $(t \gg 1 / \lambda)$ probability density of the dynamical variable $X$ is Gaussian with variance equal to $\sigma_{\eta}^{2} /(2 \lambda)$. In hindsight, the observed disagreement is less surprising. $\sigma_{M}$ is the measure of spreading of statistically independent trajectories that start at $X(0)$. On the other hand, the endpoints of intervals used to calculate the relative displacements $Z(t, \tau)$ may be interpreted as the final positions of two correlated random walkers who both start at $X(0)$ and whose correlation function $\rho(t)$ is given eq. (7). Thus, $\sigma_{S}$ quantifies the spreading of such walkers and consequently describes a completely different process than $\sigma_{M}$ does. This interpretation is discussed in detail in the appendix.

Having determined the scaling properties of the OU Langevin model, we now apply DFA to the very same numerical ensembles as those in fig. 1. More specifically, we carry out linear detrended fluctuation analysis of the increments of $X(t)$ (these increments are obtained 


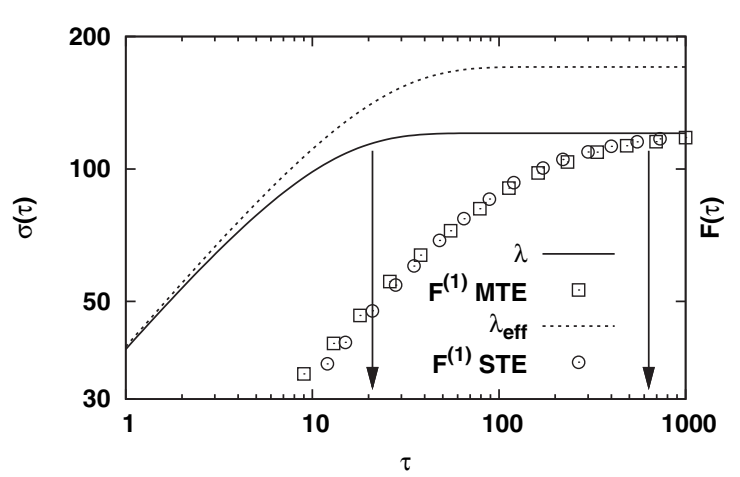

Fig. 2: Detrended fluctuation analysis of the increments of the solution $X$ of the OU Langevin equation (squares). The open circles denote the rms of DFA residuals calculated using the STE while the squares correspond to MTE. The theoretical value of the standard deviation is plotted for the MTE (solid line, cf. eq. (9)) and the STE (dashed line, cf. eq. (15)). The model's parameters are the same as in fig. 1.

by differencing the time series of numerically calculated values of $X)$. We can see in fig. 2 that, with the exception of very short segments for which the linear least-square fit is not reliable, MTE (squares) and STE (circles) averages of $F^{(1)}(\tau)$ coincide. This equivalency is obviously absent for the ordinary standard deviation. Thus, it must be brought about by detrending. Let us revisit eq. (12). For a fixed value of $t$, or in other words while calculating the variance of $Z(t, \tau)$ as a function of $\tau$, the first term on the r.h.s. of this equation is stochastic, while the second one is purely deterministic. Consequently, their covariance vanishes. Thus, the partitioning of a single trajectory characteristic of DFA is equivalent to building up trends that satisfy the "trend + signal" decomposition assumption eq. (3). As long as the investigated time series may be decomposed in the very same way, DFA is the robust scaling detection algorithm.

We mentioned that DFA adopts the very specific model of non-stationarity given by eq. (3) that consists of polynomial trends superposed on the signal. In this case, trends are the source of non-stationarity. However, it is not true that every non-stationary time series must be of that kind. Although one can always arbitrarily carry out such decomposition, the resulting terms need not be statistically independent and therefore need not have a null covariance. What is even more important, nonvanishing covariance may not be related to the type of non-stationarities induced by the environment but to the very nature of the dynamics whose scaling properties we are investigating. One can see in fig. 2 that the growth of the rms of DFA residuals $F^{(1)}(\tau)$ with time does not follow that of the standard deviation of $X(t)$ for either the MTE (cf. eq. (9)) or the STE (cf. eq. (15)). The values of $F^{(1)}(\tau)$ are significantly smaller than both theoretical curves. This in itself is not surprising taking into account that $F^{(1)}(\tau)$ is the rms of detrended fluctuations. However, as indicated by the two vertical arrows in this figure, linear detrending

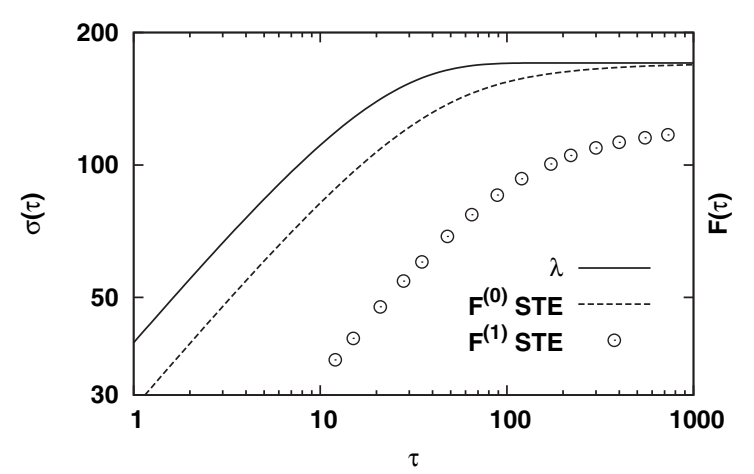

Fig. 3: The comparison of zeroth (dashed line) and first (circles) order DFA applied to the increments of the solution $X$ eq. (8) $\left(\lambda=0.055\right.$ and $\left.\sigma_{\eta}=40\right)$. Equation (16) was used to calculate the dependence of $F_{S}^{(0)}$ on the length $\tau$ of the data window. The rms value of the linear DFA residuals $F_{S}^{(1)}$ was numerically calculated by partitioning a single trajectory into non-overlapping windows of length $\tau$. The solid line represents the theoretical value of the standard deviation for the STE, cf. eq. (15).

delays the approach of $F^{(1)}(\tau)$ to its asymptotic value by at least a decade $\left(\sigma_{M}\right.$ reaches $95 \%$ of the asymptotic value $\sqrt{\sigma_{\eta}^{2} / 2 \lambda}=120.605$ at $\tau=22$ while $F^{(1)}(\tau)$ reaches the same value at $\tau=635$ ).

The polynomial detrending characteristic of DFA may not be the only source of discrepancy between standard deviation and rms of residuals shown in fig. 2. Regardless of the type of ensemble averaging, the calculation of the ordinary standard deviation involves only the endpoints of intervals of given length $\tau$. On the other hand, $F^{(m)}(\tau)$ is calculated using all $\tau+1$ values of detrended fluctuations in such intervals. For the OU Langevin model, it is possible to calculate the following STE average

$$
\left[F_{S}^{(0)}(t)\right]^{2}=\frac{1}{t} \int_{0}^{t} \sigma_{S}^{2}\left(t^{\prime}\right) \mathrm{d} t^{\prime}=\frac{\sigma_{\eta}^{2}}{\lambda^{2} t}\left(\lambda t+e^{-\lambda t}-1\right) .
$$

$F_{S}^{(0)}(t)$ may be interpreted as the zeroth-order DFA. The power series expansion for $F_{S}^{(0)}(t)$ about $t=0$ yields the scaling index $1 / 2$ which is equal to that of the standard deviation. In fig. 3 we compare the dependence of $\sigma_{S}$, $F_{S}^{(0)}$ and $F_{S}^{(1)}$ on the length $\tau$ of data segments. It follows from eq. (15) that $\sigma_{S}$ reaches $95 \%$ of the asymptotic value $\sqrt{\sigma^{2} / \lambda}=170.561$ at $\tau=43$ while $F^{(0)}(\tau)$ reaches the same value at $\tau=187$. As we already pointed out during the analysis of fig. $2, F^{(1)}(\tau)$ crosses the chosen threshold value at $\tau=635$. Thus, the slow approach of $F_{S}^{(1)}(\tau)$ to its asymptotic value is caused predominately by the linear detrending.

A priori knowledge of the scaling properties of the OU Langevin model guides our choice of the least-square fitting interval used in the determination of the shorttime scaling exponent. For sufficiently short time intervals, the slope of $F^{(1)}(\tau)$ approaches the expected value of $1 / 2$. 
However, such guidance is rarely available for physiological data, and consequently the choice of time intervals is ambiguous. In the worst-case scenario, the slow growth of $F^{(m)}(\tau)$ may be easily misinterpreted as a pseudoscaling region, especially when the DFA is performed on very short data segments. This effect along with the problem of applicability of the Langevin equation to EEG modeling are discussed in [23].

At the beginning of the paper we asked whether DFA is applicable in the short-time limit. The answer to this question is yes. The answer to the second question concerning the validity of the "trend + signal" decomposition comes in two parts. Havlin et al. [24] while reviewing the application of statistical physics methods to cardiac diagnostic wrote: "The DFA analysis yields a crossover point for the fractal slope for the scale $m=4$. It should be noted, however, that the crossover point in the DFA analysis is not a sharply defined point, rather the change in fractal slope takes place in a gradual way." The analysis of the OU Langevin model discussed herein suggests that the failure of the decomposition assumption underlies this gradual crossover at intermediate times. The implications of this failure concern not only the interpretation of short-time dynamics of physiological time series but also the determination of the values of scaling exponents which may be used as clinical diagnostic parameters. As for the second part of the answer, we demonstrated that the partitioning of a single trajectory amounts to building up deterministic trends. Consequently, while the MTE and STE estimates of ordinary standard deviation are different, the rms of DFA residuals is not affected by the method of averaging. Thus, we discovered quite an unexpected property of DFA. The equivalency of MTE and STE averages for stationary processes was postulated as early as 1945 by Wang and Uhlenbeck [21]. Herein we demonstrated that this assumption may be false even for a stationary and ergodic OU Langevin equation which is the simplest possible model of a physiological control system. Moreover, the latest theoretical and experimental studies [25-30] suggest that nonergodicity may be the rule rather than exception. Consequently, the validity of phenomenological (STE) ensembles, tacitly assumed in most biomedical and physiological applications, should be revisited.

\section{$* * *$}

MI thanks the U.S. Army Research Office for support of this research. We thank a referee for suggesting the twowalker interpretation of STE averaging.

\section{ApPENDIX}

One interpretation of the STE averaging is to consider two random walkers $X_{1}$ and $X_{2}$ that both start at $X(0)=0$ and are correlated $\rho(\tau)=\exp (-\lambda \tau)$. The final positions of such walkers may be interpreted as the endpoints of intervals used to calculate the relative displacements
$Z(t, \tau)$. The joint probability density $P\left(X_{1} X_{2} \tau\right)$ for such walkers is given by eq. (44) in Wang and Uhlenbeck [21]:

$$
P\left(X_{1} X_{2} \tau\right)=\frac{\lambda}{\pi \sigma_{\eta}^{2} \sqrt{1-\rho^{2}}} \exp \left[\frac{-\lambda}{\sigma_{\eta}^{2}} \frac{X_{1}^{2}+X_{2}^{2}-2 \rho X_{1} X_{2}}{1-\rho^{2}}\right]
$$

The distribution of the distance $Q=X_{1}-X_{2}$ is determined by integrating over one of the variables to obtain

$$
P(Q, \tau)=\sqrt{\frac{\lambda}{2 \pi[1-\rho(\tau)] \sigma_{\eta}^{2}}} \exp \left[\frac{-\lambda}{2 \sigma_{\eta}^{2}} \frac{Q^{2}}{1-\rho(\tau)}\right] .
$$

The mean square displacement of the distance is therefore

$$
E\left[Q^{2}(\tau)\right]=\int_{-\infty}^{\infty} Q^{2} P(Q, \tau) \mathrm{d} Q=\frac{\sigma_{\eta}^{2}}{\lambda}[1-\rho(\tau)],
$$

which is the same as eq. (15). The STE refers to the spreading in time of the distance between two correlated random walkers. On the other hand, in MTE the spreading concerns the ensemble of statistically independent trajectories. Consequently, the MTE and STE averages do not correspond to the same dynamical process as was assumed historically.

\section{REFERENCES}

[1] Peng C. K., Buldyrev S. V., Havlin S., Simons M., Stanley H. E. and Goldberger A. L., Phys. Rev. E, 49 (1994) 1685.

[2] Bashan A., Bartsch R., Kantelhardt J. W. and Havlin S., Physica A, 387 (2008) 5080.

[3] Mandelbrot B., Phys. Scr., 32 (1985) 257.

[4] Eke A., Herman P., Kocsis L. and Kozak L. R., Physiol. Meas., 23 (2002) R1.

[5] Peng C.-K., Havlin S., Stanley H. E. and GoldBerger A. L., Chaos, 5 (1995) 82.

[6] Huikuri H. V., Makikallio T. H., Peng C. K., GoldBerger A. L., Hintze U. and Moller M., Circulation, 101 (2000) 47.

[7] Beckers F., Verheyden B. and Aubert A. E., Am. J. Physiol. Heart Circ. Physiol., 290 (2006) H2560, http://dx.doi.org/10.1152/ajpheart.00903.2005.

[8] Mourot L., Bouhaddi M., Gandelin E., Cappelle S., Nguyen N. U., Wolf J.-P., Roulllon J. D., Hughson R. and Regnard J., Auton. Neurosci., 137 (2007) 27, http//dx.doi.org/10.1016/j.autneu.2007.06.284.

[9] PCh I., Bunde A., Amaral L. A., Havlin S., FritschYelle J., Baevsky R. M., Stanley H. E. and GoldBERGER A. L., Europhys Lett., 48 (1999) 594.

[10] Tulppo M. P., Hughson R. L., Maekikallio T. H., Airaksinen K. E., Seppaenen T. and Huikuri H. V., Am. J. Physiol. Heart Circ. Physiol., 280 (2001) H1081.

[11] Maekikallio T. H., Koistinen J., Jordaens L., Tulppo M. P., Wood N., Golosarsky B., Peng C. K., Goldberger A. L. and Huikuri H. V., Am. J. Cardiol., 83 (1999) 880. 
[12] Castiglioni P., Parati G., Civijian A., Quintin L. and Rienzo M. D., IEEE Trans. Biomed. Eng., 56 (2009) 675, http//dx.doi.org/10.1109/TBME. 2008. 2005949.

[13] Beckers F., Verheyden B., Ramaekers D., Swynghedauw B. and Aubert A. E., Clin. Exp. Pharmacol. Physiol., 33 (2006) 431, http//dx.doi.org/ $10.1111 / \mathrm{j} .1440-1681.2006 .04384 . \mathrm{x}$.

[14] Latka M., Glaubic-Latka M., Latka D. and West B., Chaos, Solitons Fractals, 20 (2004) 165.

[15] Taqqu M. S., Teverovsky V. and Willinger W., Fractals, 3 (1995) 785.

[16] Goldberger A. L., Amaral L. A., Glass L., Hausdorff J. M., Ivanov P. C., Mark R. G., Mietus J. E., Moody G. B., Peng C. K. and Stanley H. E., Circulation, 101 (2000) E215.

[17] Feder J., Fractals (Plenum Press, New York) 1988.

[18] Bassingthwaighte J., Liebovitch L. and West B., Fractal Physiology (Oxford University Press) 1994.

[19] West B. J., Where Medicine Went Wrong: Rediscovering the Path to Complexity (World Scientific Publ. Co. Inc.) 2006.

[20] Lindenberg K. and West B. J., The Nonequilibrium Statistical Mechanics of Open and Closed Systems (VCH, New York) 1990.
[21] Wang M. and Uhlenbeck G., Rev. Mod. Phys., 17 (1945) 323.

[22] Hastings H., Sugihara G., Hartings H. and Hastings H., Fractals - A User's Guide for the Natural Sciences (Oxford University Press, Oxford) 2002.

[23] Ignaccolo M., Latka M., JernajczyK W., Grigolini P. and West B. J., Phys. Rev. E, 81 (2010) 031909.

[24] Havlin S., Amaral L. A., Ashkenazy Y., Goldberger A. L., PCh I., Peng C. K. and Stanley H. E., Physica A, 274 (1999) 99.

[25] McCauley J. L., Physica A, 387 (2008) 5518.

[26] Lubelski A., Sokolov I. M. and Klafter J., Phys. Rev. Lett., 100 (2008) 250602.

[27] Brokmann X., Hermier J.-P., Messin G., Desbiolles P., Bouchaud J.-P. and Dahan M., Phys. Rev. Lett., 90 (2003) 120601.

[28] Margolin G. and Barkai E., Phys. Rev. Lett., 94 (2005) 080601.

[29] Margolin G., Protasenko V., Kuno M. and Barkai E., J. Phys. Chem. B, 110 (2006) 19053, http//dx.doi. org/10.1021/jp061487m.

[30] Masuda N. and Ainara K., Neural Comput., 15 (2003) 1341, http//dx.doi.org/10.1162/089976603321780308. 